\title{
An Interferometer for Measuring Gradients in Both Refractive Index and Thickness of Large or Small Optics
}

\author{
J. B. Saunders \\ Institute for Basic Standards, National Bureau of Standards, Washington, D.C. 20234
}

(March 24, 1969)

\begin{abstract}
A small aperture prism interferometer is described for measuring refractive index and thickness gradients between pairs of adjacent points in both small and large optics. It is relatively free from vibration problems and thermal gradients are reduced to a minimum by confining the two component beams of light to a relatively small space. Large specimens are tested by scanning them with the light beam.
\end{abstract}

Key words: Interferometer; optical homogeneity; refractive index.

\section{Introduction}

The classical instrument for measuring variations in refractive index of glass was described by Twyman and Green [1] ${ }^{1}$ in 1916. This interferometer is quite suitable for testing small specimens but is impractical for testing specimens larger than 8 to $10 \mathrm{in}$. because of vibrations, temperature problems, and difficulties in making large aperture interferometers.

The Kösters prism interferometer was suggested for measuring the homogeneity of optical media in 1963 [2] but a description of this application has not been published. This interferometer is quite practical for measuring index gradients in both small and large optics. It is relatively free from vibration problems and thermal gradients are reduced to a minimum by confining the two component beams of light to a relatively small space. Large specimens are tested by scanning them with the light beam.

\section{Components of the Interferometer}

Figure 1 shows the optics of a modified Kösters prism interferometer. The light source may be either a continuous emission laser or a highly monochromatic spectral source, such as Krypton or Mercury 198. If a laser is used, a short focus (high power), single element, plano-convex lens that has its axis slightly tilted relative to the principal ray, will provide adequate divergence and sufficient freedom from unwanted interference fringes.

The Kösters prism interferometer has its two components cemented together for stabilization of several

\footnotetext{
${ }^{1}$ Figures in brackets indicate the literature references at the end of this paper.
}

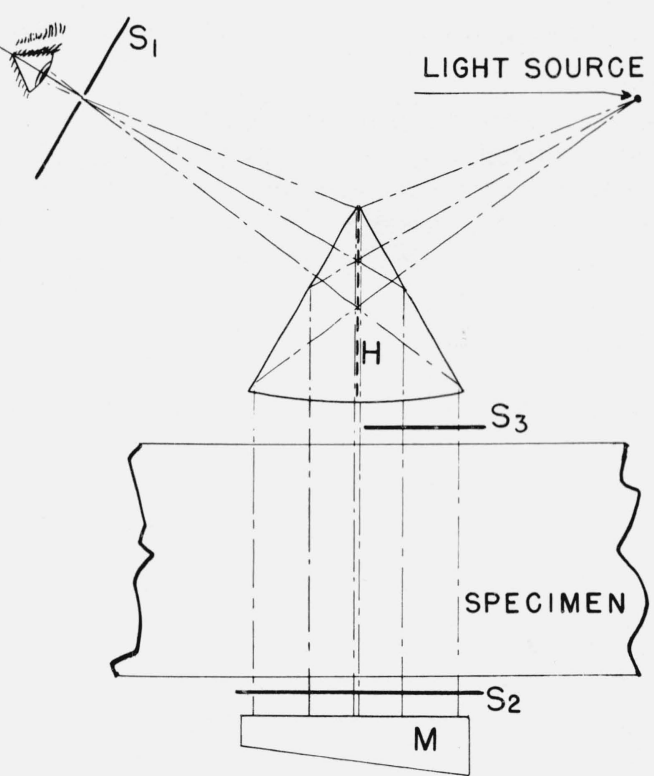

FIGURE 1. Optics of the interferometer. The figure is a section through the center of the prism.

of its interferometric adjustments. These adjustments for a built-in-wedge prism [3] are: (1) one component of the fringe width is adjusted to optimum width, and (2) the zero order of interference is adjusted to the most favorable position (the center) where it remains during further operational adjustments. The adjustments for a prism without a built-in-wedge are to set the zero order of interference at the center of the prism and to maximize its width by polishing the base. 
The two types of prisms are equally accurate but the integral parts of the orders of interference in the built-in-wedge type are easier to read.

The base surface of the prism is figured to be spherical and convex. The prism serves as: (1) a collimator lens, (2) a beam divider for dividing the beam two times, (3) two mirrors for deflecting the transmitted and reflected beams to a common direction, (4) a near perfect compensator so that white light interference can be used if desired, and (5) a collector lens.

If a laser is used for the source, the mirror, M, may be a small uncoated optical flat. However, if the mirror does not have an opaque reflecting coat, its bottom surface should either be tilted to avoid reflecting light back through the prism or it should be rough ground, or coated with something like petroleum jelly, cedar oil, or a similar fluid to reduce reflection. A sheet of black paper, soaked in the fluid and contacted to the back of the mirror, serves as an excellent nonreflection coat. The remaining components consist of the specimen and three simple screens $S_{1}, S_{2}$, and $\mathrm{S}_{3}$.

A calibrated glass wedge [4], located between the specimen and prism, enhances the accuracy of reading the orders of interference.

\section{Adjustment of the Interferometer}

The only adjustments, other than those made invariant by cementing the prism components together, are positioning the light source and mirror relative to the prism. The source should be located near the focal plane of the prismatic lens formed by the base of the prism so as to collimate the two light beams (one from each of the two prism components) in the specimen area of the interferometer. The source should also be adjusted in the plane of fig. 1 so that the two component beams (reflected and transmitted by the beam divider) are parallel to the beam dividing plane.

The distance from the prism to the mirror may be chosen at will, however, adequate space should be allowed for free movement of the thickest specimen that is intended to be tested. The angular position of the mirror about an axis perpendicular to the beam dividing plane is not critical, except that the angle of incidence of the light should remain small. The only critical adjustment that requires attention is the angular position of the mirror about an axis parallel to the vertex of the prism. This adjustment, which can be controlled by a single screw that rotates the mirror relative to the prism assembly, controls the component width of the transmission fringes (defined below). If a prism with no built-in-wedge (fringes parallel to the dividing plane) is used, this adjustment of the mirror only causes the fringes to change separation without rotation. If a built-in-wedge prism is used it causes the fringes to rotate about their points of intersection with the dividing plane.

The fringes produced by light reflected from the two surfaces of the specimen are relatively independ- ent of interferometer adjustments. If the surfaces of the specimen are adjusted to be approximately normal to the incident light, the width of the fringes depends only upon the refractive index and angle between the two reflecting surfaces of the specimen. The angle between the two surfaces of the specimen should form a 3 to $15 \mathrm{~s}$ wedge so that the two component beams reflected from them will form interference fringes of suitable widths for accurate measurements. The two surfaces should be flat to within $0.1 \mu \mathrm{m}$ per inch to avoid excessive variation in fringe width.

\section{Opaque Screens for Obstructing Unwanted Light}

Three opaque screens (thin, black cardboard is quite adequate) are used to obstruct light which would otherwise interfere with fringe visibility. The single beam of light from the source is divided into two beams by the beam divider, $\mathrm{H}$. When unobstructed by screens $S_{2}$ and $S_{3}$, each of these beams is incident on the two optical surfaces of the specimen and the mirror, M. Therefore, three components of each of the two beams return to the prism. These six beams are again divided by the beam divider into two components each. One set of six components is transmitted toward the observer and the other set is returned toward the souce. If the observer receives the six beams that are transmitted toward him he sees a complex interference pattern that is useless for the test.

A screen, $S_{1}$ (fig. 1), in the focal plane of the source that is dark on the observer's side, white on the source side and having a small round hole (approximately $1 \mathrm{~mm}$ in diameter), can be adjusted to transmit the pair of beams from $M$, if $M$ is adjusted normal to the beam divider. Thus, when the specimen is absent the observer sees a single set of interference fringes. The set of fringes produced by light from $M$ is defined as "transmission fringes" since the light that produces them is transmitted by the specimen during the test. The fringes produced by light reflected from the two surfaces of the specimen are defined as "reflection fringes."

When the specimen is inserted the four beams that are reflected from its two surfaces will each form an image of the source in the plane of $S_{1}$. If the surfaces of the specimen are approximately parallel to $M$ the four beams will pass through the aperture in $S_{1}$ and interfere with the transmission fringes. These four beams may be adjusted, by slightly tilting the specimen, to fall just outside the aperture in $S_{1}$, with no observable effect on the position of the beams from $M$ and no appreciable interference with the transmission fringes.

The reflection fringes are made visible by intercepting the light to $M$ with a screen $S_{2}$ (preferably dark on the incident side), intercepting one of the beams from the prism with another screen $S_{3}$ and removing $S_{1}$ from the beam. This leaves only two beams reflected from the specimen to form the reflection fringes. 


\section{Movements of the Specimen}

The specimen rests on a saddle that can be moved along a straight line normal to the dividing plane, along a set of ways, without rotation. The set of ways should have a scale attached to it that permits the sample to be moved equal intervals. A fixed reference point, $\mathrm{P}_{1}$ (fig. 2), should be located between the prism

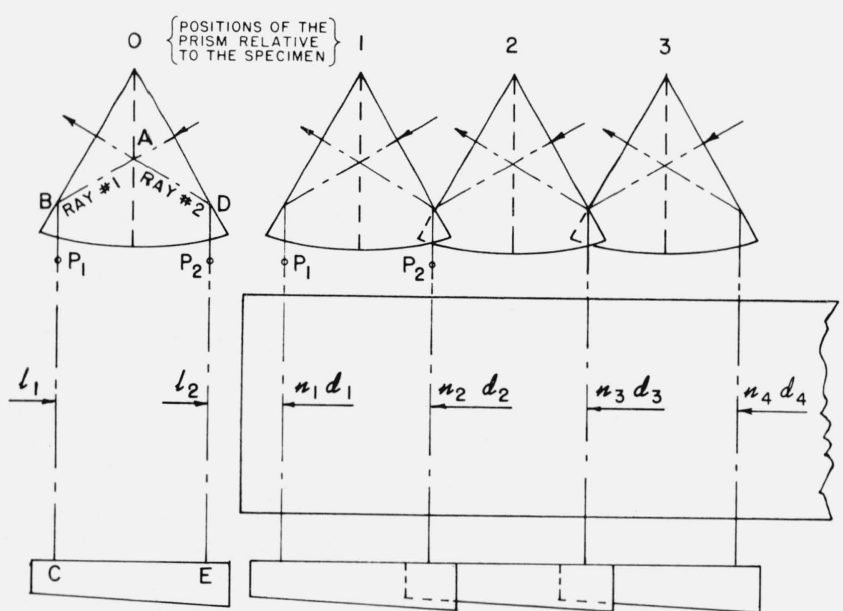

FIGURE 2. Several positions of the specimen relative to the prism. Observations are made without the specimen and with the specimen located at each of several positions relative to the prism.

and specimen, in the beam that forms the reflection fringes. A second reference point, $\mathrm{P}_{2}$, symmetrical to $\mathrm{P}_{1}$, with respect to the beam divider, will insure good visibility of the reference points in the transmission fringes. The separation of these two points equals the interval of movement of the specimen between observations as it traverses the light beam. These two reference points may be small beads on a fine wire attached to the prism cell and stretched normally to the beam dividing plane.

Data can be taken along any straight or curved path across the specimen as long as ray 1 passes through the specimen in exactly the same place that ray 2 passed through before the specimen was moved from the previous position. The number of positions at which measurements are made depends upon the length of the sample and the separation of the reference points. The separation of the reference points may be any value from near zero to slightly less than the aperture of the prism. The prisms used for these tests had diameters of $3 \mathrm{~cm}$.

Figure 2 shows several positions of the prism relative to the sample; although it is the sample that is moved relative to the prism. The prism, light source, reference points, and mirror are rigidly connected to insure interferometric stability. If we let the two optical paths, "ABC" and "ADE", be represented by $l_{1}$ and $l_{2}$, respectively, the order of interference, $Q_{0}$, observed at the superimposed reference points, $P_{1}$ and $P_{2}$ will be given by the equation,

$$
Q_{0}=2\left(l_{2}-l_{1}\right) / \lambda .
$$

The adjustment for $Q_{0}$ is controlled by the single screw adjustment mentioned above. This adjustment should not be altered during the test.

If, when the prism is in any position $\nu(\nu=1,2 \ldots)$, we let $T_{\nu}$ and $R_{\nu}$ be observed orders of interference for the transmission and reflection fringes, respectively, and letting $n_{\nu}$ and $d_{\nu}$ be respectively the refractive index and thickness of the sample at the place where ray 1 traverses it, then it can be shown that:

$$
\begin{aligned}
& T_{\nu}=Q_{0}+\left[d_{\nu+1}\left(n_{\nu+1}-1\right)-d_{\nu}\left(n_{\nu}-1\right)\right] 2 / \lambda, \\
& \Delta R_{\nu} \equiv R_{\nu+1}-R_{\nu}=\left[d_{\nu+1} n_{\nu+1}-d_{\nu} n_{\nu}\right] 2 / \lambda .
\end{aligned}
$$

It will be noted that the final position of the specimen is required for evaluating $R_{\nu}$ but not $T_{\nu}$.

If $n$ and $d$ be the nominal refractive index and thickness of the sample, respectively, in the direction traversed by the light, $\Delta n_{\nu}$ and $\Delta d_{\nu}$ the variations in $n$ and $d$ between the two interfering beams (rays 1 and 2), it can be shown that [5], to the approximation aimed at in this analysis

$$
\begin{aligned}
\Delta n_{\nu} & =\left[n\left(T_{\nu}-Q_{0}\right)-(n-1) \Delta R_{\nu}\right] \lambda / 2 d, \\
\Delta d_{\nu} & =\left[\Delta R_{\nu}-T_{\nu}+Q_{0}\right] \lambda / 2 .
\end{aligned}
$$

The index gradient and wedge (angle between the two surfaces) of the specimen are obtained by dividing $\Delta n_{\nu}$ and $\Delta d_{\nu}$, respectively, by the separation of the reference points. The total changes in index and thickness are obtained by adding the increments $\Delta n_{\nu}$ and $\Delta d_{\nu}$, respectively. It can be shown that, to the same approximation mentioned above,

$$
\begin{aligned}
& \sum_{\nu=1}^{\nu} \Delta n_{\nu}=n_{\nu+1}-n_{1} \\
&= {\left[n\left(\sum_{\nu=1}^{\nu} T_{\nu}-\nu Q_{0}\right)-(n-1) \sum_{\nu=1}^{\nu} \Delta R_{\nu}\right] \lambda / 2 d, } \\
& \sum_{\nu=1}^{\nu} \Delta d_{\nu}=d_{\nu+1}-d_{1} \\
&=\left[\sum_{\nu=1}^{\nu} \Delta R_{\nu}-\sum_{\nu=1}^{\nu} T_{\nu}+\nu Q_{0}\right] \lambda / 2 .
\end{aligned}
$$

It is sometimes important for specifications to know the maximum variation in the refractive index and thickness of large optics. When values for $\left(n_{\nu+1}-n_{1}\right)$ and $\left(d_{\nu+1}-d_{1}\right)$ are tabulated they show the maximum and minimum values, from which maximum differences are readily obtainable. Obviously the results apply only to that part of the specimen covered by the selected reference points and may not include the maximum variations of the entire specimen unless the points are chosen to coincide with them. 


\section{Reduction of Error and Elimination of $Q_{0}$}

Since the wavelength, $\lambda$, the refractive index, $n$, and thickness, $d$, of the specimen are usually known more accurately than the orders of interference, the errors in the determined values of $\Delta n_{\nu}$ and $\Delta d_{\nu}$ arise almost entirely from the errors in the measured values of the orders of interference. This, of course, is based on the assumption that temperature gradients remain negligible.

It is apparent from eqs (3) and (4) that an error in $Q_{0}$ will produce errors in $\left(n_{\nu+1}-n_{1}\right)$ and $\left(d_{\nu+1}-d_{1}\right)$ that are $\nu$ times the corresponding errors in $\Delta n_{\nu}$ and $\Delta d_{\nu}$. This error can accumulate to relatively large values when observations are made at many points across a large specimen. On the other hand, the error in $\Delta R_{\nu}$ does not accumulate since it can never exceed twice the maximum error of a single observation. It can also be shown that all biased errors in $Q_{0}, R_{\nu}$, and $T_{\nu}$ are automatically eliminated by differences. The result of random errors in all observations are obviously reduced by averaging the results of observations taken at the same points during two traversals of the specimen. If the specimen is rotated $180^{\circ}$ between the two traversals and observations are made at the same points but in reverse order, the quantity $Q_{0}$ does not affect the average and, therefore, may be eliminated from the observations.

If the two traversals are indicated by subscripts of parenthesis, eq (1) becomes, for the first and second traversal, respectively,

$$
\left.\begin{array}{l}
\left(\Delta n_{\nu}\right)_{1}=\left[n\left(T_{\nu}\right)_{1}-n\left(Q_{0}\right)_{1}-(n-1)\left(\Delta R_{\nu}\right)_{1}\right] \lambda / 2 d, \\
\left(\Delta n_{\nu}\right)_{2}=\left[n\left(T_{\nu}\right)_{2}-n\left(Q_{0}\right)_{2}-(n-1)\left(\Delta R_{\nu}\right)_{2}\right] \lambda / 2 d .
\end{array}\right\}
$$

If no adjustment of the prism, relative to the mirror, is made during the observations then $\left(Q_{0}\right)_{1}=\left(Q_{0}\right)_{2}=$ $Q_{0}$. The constancy of $Q_{0}$ is assured by the stability of this instrument. The reversal of the specimen reverses the optical and geometrical wedges between all pairs of adjacent points with the result that values for $\left(\Delta n_{\nu}\right)_{1}$ and $\left(\Delta d_{\nu}\right)_{1}$ will be identical in magnitude and of opposite sign (except for errors) to $\left(\Delta n_{\nu}\right)_{2}$ and $\left(\Delta d_{\nu}\right)_{2}$, respectively. The best values for $\Delta n_{\nu}$ and $\Delta d_{\nu}$ are, therefore,

$$
\begin{aligned}
& \overline{\Delta n_{\nu}}=\frac{1}{2}\left[\left(\Delta n_{\nu}\right)_{1}-\left(\Delta n_{\nu}\right)_{2}\right] \\
& =\left\{n\left[\left(T_{\nu}\right)_{1}-\left(T_{\nu}\right)_{2}\right]-(n-1)\left[\left(\Delta R_{\nu}\right)_{1}-\left(\Delta R_{\nu}\right)_{2}\right]\right\} \lambda / 4 d .
\end{aligned}
$$

$$
\begin{gathered}
\overline{\Delta \bar{d}_{\nu}}=\frac{1}{2}\left[\left(\Delta d_{\nu}\right)_{1}-\left(\Delta d_{\nu}\right)_{2}\right] \\
=\left\{\left(\Delta R_{\nu}\right)_{1}-\left(\Delta R_{\nu}\right)_{2}-\left(T_{\nu}\right)_{1}+\left(T_{\nu}\right)_{2}\right\} \lambda / 4 .
\end{gathered}
$$

The corresponding values for $\left(n_{\nu+1}-n_{1}\right)$ and $\left(d_{\nu+1}-d_{1}\right)$ are

and

$$
n_{\nu+1}-n_{1}=\sum_{\nu=1}^{\nu} \bar{\Delta} \overline{n_{\nu}}
$$

$$
d_{\nu+1}-d_{1}=\sum_{\nu=1}^{\nu} \overline{\Delta d}_{\nu}
$$

Care must be exercised to insure that the values obtained for $T_{\nu}$ and $\Delta R_{\nu}$ in eqs (6) and (7) represent values for the same pairs of points on the specimen. Positive identification is enhanced by the fact that $\left(\Delta R_{\nu}\right)_{1}$ and $\left(\Delta R_{\nu}\right)_{2}$ will be equal in magnitude and of opposite sign (except for errors) and the algebraic sum of $\left(T_{\nu}\right)_{1}$ and $\left(T_{\nu}\right)_{2}$ will be equal to a constant. The value of this constant is $2 Q_{0}$, which need not be determined.

\section{References}

[1] Twyman and Green, British Patent 103832.

[2] Saunders, J. B., Large aperture interferometers with small beam dividers, J. Res. NBS 67c (Eng. and Instr.), No. 3, 205 (1963).

[3] Saunders, J. B., Parallel testing interferometer, J. Res. NBS 61, 491 (1958) RP2917.

[4] Technical News Bulletin NBS 42, No. 2, 33 (1958).

[5] Candler, C., Modern Interferometers, p. 143 (Hilger \& Watts Ltd., Hilger Div., London, England (1951).

(Paper 73C 1\&2-283) 